\title{
Geophysics in Near Surface Investigations
}

\author{
PREFACE TO THE SPECIAL ISSUE
}

\begin{abstract}
Jadwiga JARZYNA
AGH University of Science and Technology

Faculty of Geology Geophysics and Environmental Protection, Kraków, Poland e-mail: jarzyna@uci.agh.edu.pl
\end{abstract}

The increasing demand of human society for sustainable development and preserving natural environment in good condition forces modification of engineering technologies and methods of environmental protection. Environmental and engineering geophysics, the youngest branch of geophysics, brings an answer to still actual questions of how to get information and collect current data on the environment status. The non-invasive, non-destructive and low-cost geophysical technologies are now able to provide 3D and 4D representations of underground rock formations, including media that fill-in the pore space. So, the applied geophysics notices its chance and dynamically develops the methods and methodologies for new applications. Improved technologies and new, special methods in applied geophysics are now addressed not only to the traditional clients of prospecting geophysics but also to near surface investments.

Environmental and engineering geophysics covers a broad area of applications, including cartography of shallow geological formations and prospecting for not deeply situated raw materials and water investigations, monitoring quantities being the indicators of anthropogenic devastation of environment, measuring the parameters essential for civil engineering and archaeology. Geophysical recording of salinity of underground waters, the level of radioactivity or changes of soil parameters due to industrial activity goes without saying. 
Conferences, scientific meetings, and technical presentations of equipment for shallow geophysics have taken place all over the world for a long time. Groups of specialists who develop methods and try to find new areas of application of geophysics in the near surface investigations are still growing. Near Surface2008 - the $14^{\text {th }}$ European Meeting of Environmental and Engineering Geophysics held in Cracow, Poland, on 15-17 September 2008 is one of the meaningful events in the world of applied geophysics. Papers presented at the conference and discussion meetings during it gave a background to prepare the special issue of Acta Geophysica devoted to problems of near surface geophysical investigations.

In this special issue of Acta Geophysica, entitled "Geophysics in near surface investigations", several problems that can be solved by shallow geophysical technologies are discussed; the issue contains also some papers on applied geophysics submitted to this journal as regular ones. Let me briefly outline the main topics of the contributions collected in this volume.

High resolution seismics (HRS) in sulphur in situ mining gives detailed information on the structure and tectonics of deposits and overburden strata. The range of subsidence provoked by sulphur well mining causing technical difficulties during exploitation can be estimated on the basis of the comparison of seismic sections before and during exploitation. Such a methodology enables to determine processes of changing structure of the deposit and overburden strata.

Experimental high resolution seismic surveys conducted in the site belonging to the salt basin detect underground salt cavities at a depth of about hundred meters. After the end of mining operations, salt cavities originally situated in salt layer progress into the overburden and are situated in the immediate proximity of hard, dolomite layer. Set of microcracks in the dolomite is observed. There is a serious fear of a collapse on the surface. HRS turns out to be efficient in detecting cavities, and an accuracy of estimation of horizontal extensions of cavities is surprisingly good.

Acoustic full waveforms recorded in wells are the simplest way to get velocity of $P$ wave, $S$ wave and Stoneley wave in situ. At shallow depths, in soft formations, some difficulties arise with a proper evaluation of the $S$-wave velocity due to the lack of refracted $S$ wave in acoustic full waveform in case when its velocity is lower than the velocity of mud. Dynamic approach to selection of a proper value of semblance to determine the proper slowness and arrival in automatic processing of acoustic full waveforms solves the problem.

Time-frequency methods, i.e., the Continuous Wavelet Transform (CWT) and the Matching Pursuit (MP) algorithm, are applied to acoustic full waveform processing for the precise identification of selected acoustic waves, waveform decomposition into separate waves, and determination of zones of different elastic parameters in geological profiles. CWT is used to improve 
qualitative interpretation, and time-depth-frequency plots for a given frequency are constructed to distinguish the waves and identify gas-bearing zones. MP provides a better resolution in time-frequency space, and thus is used to extract individual waves from the acoustic waveform. For the extracted waves, slowness and velocity are calculated.

Potential alluvial water saturated formations are recognized by a few geophysical acquisitions, including ground penetrating radar, DC resistivity prospecting, seismic refraction survey, and magnetic resonance soundings. Two wells are drilled and the lithology and water flow rate are acquired from them. Water height in the aquifer, effective porosity, water content, permeability and transmissivity of the alluvial deposits are determined on the basis of geophysical measurements.

Moisture ingress is one of the major damaging factors for masonry buildings. Non-destructive application of complex resistivity (CR) measurements sensitive to textural properties as well as to pore fluid chemistry of wet porous media provides helpful information for conservators. A comprehensive laboratory study supports CR in distinguishing between level of salinity and saturation degree. Combination of complex resistivity tomography and GPR in reflection and transmission configuration in a field-scale flooding experiment shows some unexpected differences giving new information about building state and the influence of salinity on travel time of electromagnetic waves and their attenuation.

Combining the interpretation of disparate data sets is one of the ways to improve the information content of a set of field data. Joint inversion for electromagnetic and direct current resistivity methods enables passing over an inherent equivalence problem. Synthetic data from vertical electrical sounding and horizontal coplanar low-frequency induction sounding are inverted individually and jointly over different types of 1D earth structures. Global optimization with Monte Carlo multi start algorithm is used in the calculations. Joint inversion significantly improves the solution, reducing the ambiguity of the models.

Several methods are proposed to estimate dynamic stability of soils. One more approach, as discussed here, is based on laboratory cyclic testing. Specific features of examined soils and different conditions of static and dynamic loading are taken into account in the tests. Soil deformation under vibrodynamic loads can also be evaluated on an energy approach. Critical amount of energy dissipated by soil per unit volume is a reliable measure of deformation even in low dynamic stress range. The proposed approaches are applied to evaluate additional settlements of structures founded on the bases of different rock profile and under various static and dynamic loading conditions.

Neural network inversion of self potential anomalies, and the modular algorithm in particular, is applied for estimating the parameters of different 
simple geometrical bodies. In order to reduce the number of parameters and minimize the ambiguity in the inverted models, the Hilbert transforms are involved to determine the origin location. The inversion is tested on synthetic data from different models. An analysis of results, including Gaussian noise, is also performed with acceptable accuracy. A comparable and acceptable agreement for the published real field SP data taken from the southern Bavarian Woods confirms the results of inversion derived by the neural network.

Laboratory experimental studies of the direct current acting on models of sedimentary rocks saturated with water or hydrocarbons show that DC induces the acoustic emission. The amplitude-frequency parameters of the $\mathrm{AE}$ signals depend on the petrophysical properties of the solid phase, the salinity of porous medium, and the intensity and voltage of the electric field. Full identification of the characteristics of the AE signals generated by rocks in a DC electric field gives additional information on reservoir rocks. 